Rev. Elev. Méd. Vét. Pays trop., 1965, 18, 4 (395-403).

\title{
Première note sur quelques endoparasites des animaux sauvages de Haute-Casamance (Sénégal)
}

\author{
par S. GRETILLAT (1) ef M. GAILLARD (2)
}

\begin{abstract}
RÉSUMÉ
L'étude d'un premier lot de parasites internes (Nematoda et Pentostoma) récoltés à l'autopsie de mammifères, oiseaux et reptiles de Haute-Casamance (Sénégal) a permis de signaler:

Chez Voronus niloticus : Hastospiculum macrophalios (Parona, 1889)

: Tanqua tiara (o. v. Linstow, 1879)

Chez Coracias naevius et C. abys- : Un Squomofilaria qui pourrait appartenir sinicus : ì l'espèce S. coronoto (Rud. 1809).

Chez Meljerax metabates (faucon) : Un Thelozia localisé à l'œil.

Chez Erythrocebus potas Schreber : Streptopharoqus pigmentatus (o. v. Linsiow, : 1897).

Chez un singe vert : Protospirura muricola Gedoelst, 1918.

Cercopithecus oethiopis var, sabo- : eus (L.) et sur Syivicopra grim- : mio

Chez Cercopithecus aethiops : Les formes nymphales de. Nettorhynchus var. soboeus et Ichneumia : (Armillifer) armillatus (Wyman, 1845) aibicauda Cuv. (mangouste à : Pentastoma. queve blanche).

Un second lot de nématodes comportant notamment un certain nombre de Physoloptero parasites de l'estomac des singes et des petits carnivores ainsi que des filaires d'oseaux ef de mammifères appartenant aux genres Diplotrigena Ralllief et Henry, 1909, Setarid Wiborg, 1795, Dicrofilaria Railliet et Henry, 1911 est en cours d'étude et fera l'objet d'une nate ultérieure.
\end{abstract}

La Haute-Casamance (Sénégal) a un climat et une flore intermédiaire entre la savane guinéenne supérieure et la savane soudanaise.

Une pluviométrie relativement élevée par rapport à celle du Nord Sénégal, 1.000 à $1.300 \mathrm{~mm}$

(1) Chef du Service de Parasitologie au Laboratoire national de Recherches vétérinaıres du Sénégal, Dakar.

(2) Chef du Secteur des Grandes Endémies, Kolda, Haute-Casamance (Sénégal). répartıs sur six mois à Kolda, avec deux saisons nettement tranchées, dan's une contrée sans relief appréciable où les seuls cours d'eau. drainant d'ailleurs assez mal le terrain, sont le Sangroungou et la Casamance avec quelques marigots affluents de sa rive gauche, donne à cette partie du Sénégal, une physionomie un peu particulière.

Les cours d'eau sont bordés par une forêt galerie peu épaisse et peu profonde, alors qu'ail- 
leurs le sol gris ou rouge, sablo-argileux, recouvrant des plaques latéritiques, est occupé par une forêt sèche plus ou moins dense où les sousbois de bambous alternent avec des arbustes (Forêt de Bakor au N. E./E. de Kolda).

En dehors de ces îlots forestiers, la végétation se raréfie, tout d'abord en raison de l'aménagement des bas-fonds en rizières où l'on rencontre quelques peuplements de palmiers à huile et de roniers, ensuite à cause des déboisements opérés dans les zones sablonneuses pour la culture arachidière. La forêt fait alors place à une savane boisée où dominent fromagers, baobabs, manguiers et cailcedrats.

La faune de ces zones cultivées à haute densité de population est pauvre en grands mammifères sauvages mais par contre riche en petits rongeurs, petıts carnivores et oiseaux.

Par contre la forêt sèche où n'existe pratiquement aucun village et aucune culture est un refuge pour les grands et petits mammifères sauvages et les reptiles: lions, panthères, antilopes, sınges, phacochères, rongeurs, mangoustes, genettes, varans, ophidiens.

Nous avons pensé qu'il serait intéressant d'examiner et de relever la faune parasitaire interne de ces animaux sauvages vivant dans une région intermédiaire entre les types sahélien et guinéen où se cotoient des espèces de savane ef de grande forêt.

La présente note est l'exposé des premiers résultats obtenus à l'examen des parasites récol-. tés à l'autopsie d'animaux sauvages abattus par l'un de nous dans la région de Kolda (forêt de Bakor et savane arborée de la rive droite de la Casamance).

\section{SUPERFAMILLE DES SPIRUROIDEA RAILLIET ef HENRY, 1915}

Famille des spiruridae OERLEY, 1885

Sous-Famille des Spiroxynae BAYLIS et LANE, 1920.

Genre : Protospirura SAURAT, 1914.

Espèce : Protospirura muricola GEDOELST, 1918.

5 mâles et 5 femelles dans l'estomac d'un «Cercopithecus aethiops var. sabaeus (L.) (singe vert ou grivet) $»$.

Le matériel examiné est en excellent état de conservation.
Mâle (Fig. 1, 2, 3). Cuticule finement striée transversalement.

Longueur : 3,25 d̀ $4 \mathrm{~cm}$.

Largeur maximum : $280 \mu$.

Pharynx : $120 \mu$.

Distance anneau nerveux/extrémité céphalique : $375 / 385 \mu$.

Distance diéride/extrémité antérieure : 415/ $430 \mu$.

Distance pore excréteur/extrémité céphalique: $500 \mu$.

Longueur de la queue: $520 \mu$.

Spicule gauche : $525 / 535 \mu$.

Spicule droit : $320 \mu / 350 \mu$.

Gubernaculum: $125 \mu$.

L'extrémité postérieure est très recourbée, sa face ventrale est recouverte de stries langitudinales coupées de loin en loin par des dépressions transversales donnant d̀ cette surface un aspect réticulé.

II existe 4 paires de papilles cloacales bien développées et pédonculées et 5 paires de papilles postcloacales dont les trois antérieures sont nettement pédonculées alors que les deux terminales sont à peine distinctes et groupées à l'extrémité de la queve. Absence d'ailes caudales.

Sur un exemplaire, une papille cloacale impaire est placée en avant du bord antérieur du cloaque.

Le spicule gauche comporte une partie axiale bien développée surtout en partie antérieure avec une pointe mousse postérieure qui se confond avec l'expansion alaire latérale amincie.

Femelle (Fig. 4, 5, 6).

Longueur : 4 à $5,5 \mathrm{~cm}$ avec cuticule striée longitudinalement.

Largeur maximum : 315/320 $\mu$.

Vulve située vers le milieu du corps.

Queve de 580 d̀ $620 \mu$.

Cufs de taille) Longueur : 45 à $50 \mu$.

moyenne Largeur: 32 à $35 \mu$.

D'après les travaux d'ORTLEPP (1924), BAYLIS (1928) et en revoyant la description qu'a donnée GEDOELST en 1918 pour Protospirura muricola, nous pensons pouvoir rattacher nos spécimens à cette espèce.

Quelques petites différences dans la longueur des spicules du mâle, et la présence d'une papille impaire précloacale surnuméraire ne sont 


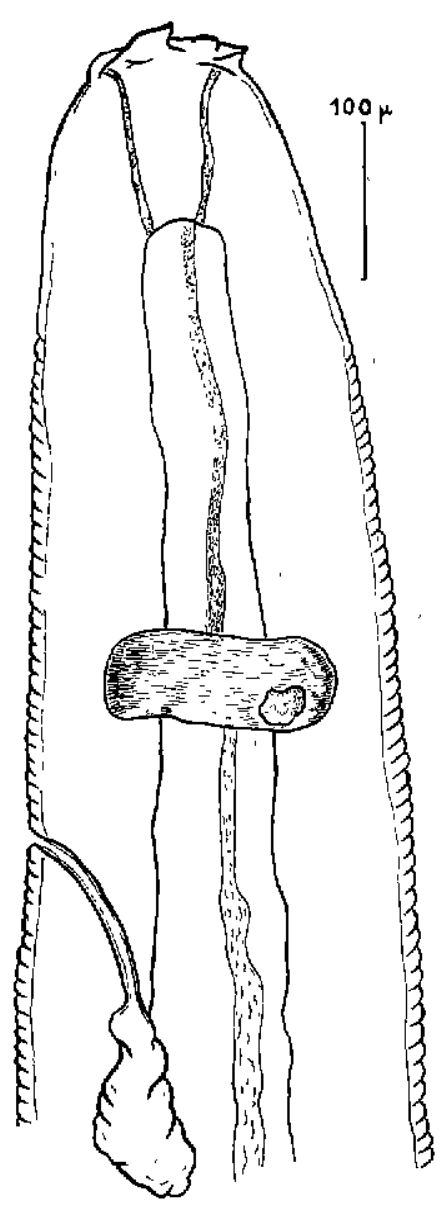

Fig. no 1. - Extrémité antérieure d'un spécimen mâle de Protospirura muricolo récollé dans l'esiomac d'un callitriche.

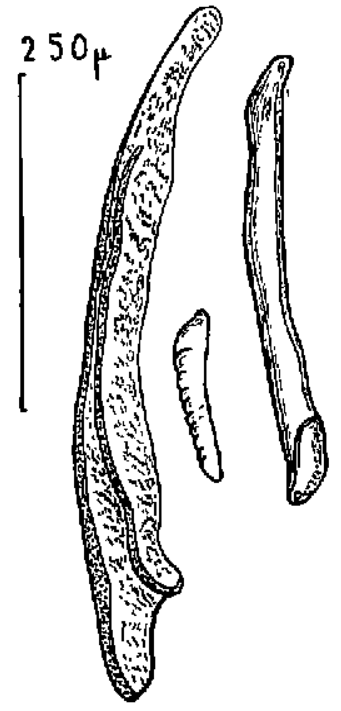

Fig. $n^{\circ} 2$. - Spicules de l'exemplaire mâle de P. muricala de la fig. 1.

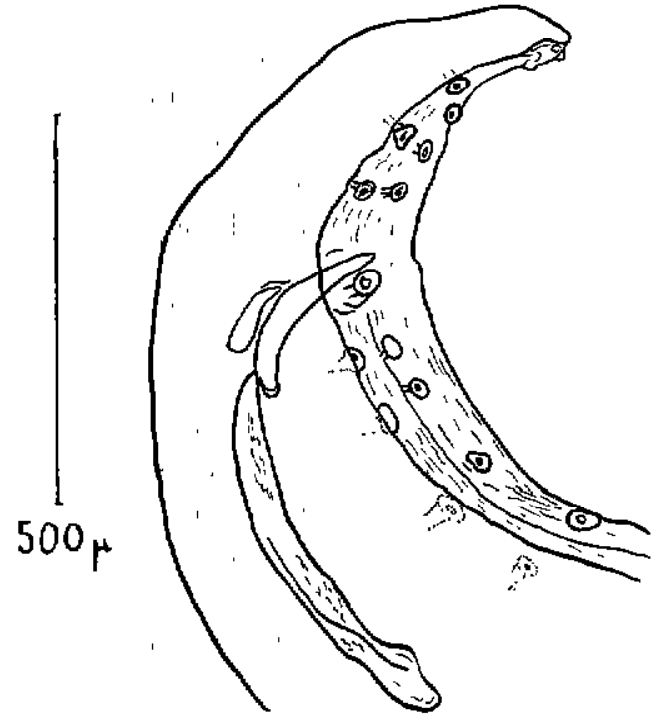

Fig. ñ3. - Extrémılé poslérieure du mâle de P. muricola de la fig. 1.

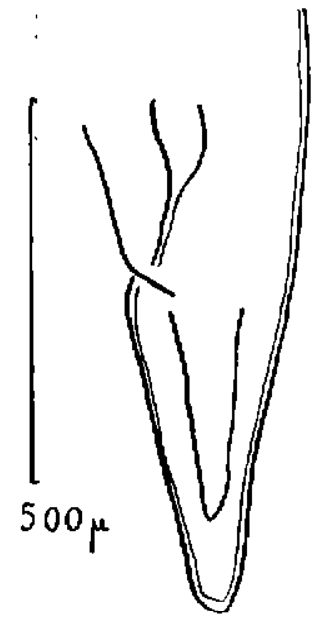

Fig. $n^{\circ}$ 4. - Extrémıté postérieure d'une des femelles de $P$. muricola récoltée dans l'estomac d'un callitriche. 
pas à notre avis des caractères suffisants pour affirmer qu'il s'agit d'une autre espèce.

Le Protospirura trouvé par FOSTER et JOHNSON en 1939 et-parasitant massivement un élevage de Cebus capucinus (singe capucin) a sensiblement la même morphologie que nos spécimens et ces auteurs n'hésitent pas à le ranger dans l'espèce $P$. muricola et admettent que son adaptation au singe auraif modifié certains de ces caractères.

D'autre part, dans un tube étiqueté Syicicapra grimmia (L.), Kolda 28-3-64, sans indication de localisation d'organe, nous avons trouvé une femelle immature de Protospirura que nous rattachons c̀ l'espèce $P$. muricola quoique l'antilope n'aie pas jusqu'à maintenant, tout au moins à notre connaissance, été mentionnée comme hôte pour cette espèce. Ce spécimen femelle a tous les caractères relevés chez les exemplaires récoltés dans l'estomac de Cercopithecus aethiops var. sabaeus (L.), avec une vulve placée en pasıtion médiane pourvue d'un ovojecteur à parois épaisses et présentant un sphincter (fig. $n^{\circ} 6$ ).

Sous-Famille des Arduenninae RAILLIET ef HENRY, 1911.

Genre Streptopharogus, BLANC, 1912.

Espèce Streptopharagus pigmentatus $(O$. von LINSTOW, 1897).

Dans l'estomac d'un Erythrocebus patas SCHREBER (Kolda 8-8-64) (singe roux) 1 femelie mûre et 2 fragments de femelles immatures.

L'exemplaire femelle renfermant des œufs, présente tous les caractères du genre Streptopharagus donné par BLANC en 1912.

Si l'on se rapporte d̀ la clé de détermination des espèces publiée par MYERS en 1954, nos spécimens appartiennent d̀ l'espèce Streptophoragus pigmentatus (O. von LINSTOW, 1897) plutôt qu'à S. baylisi ORTLEPP, 1925, tous deux parasites de primates (fig. no 7 ef 8 ).

Dimensions femelles (en millimètres)

\begin{tabular}{|c|c|c|c|}
\hline & $\frac{\text { S. baylisi }}{\text { d'après J.Myers, } 1954}$ & $\frac{\text { S.pigmentatus }}{d^{*} \text { après J.Myers, } 1954}$ & $\begin{array}{l}\text { Streptopharagus } \\
\text { in E. patas, Kolda }\end{array}$ \\
\hline $\begin{array}{l}\text { Longueur } \\
\text { Largeur } \\
\text { Pharynx L. } \\
\text { Dist. ext.: } \\
\text { cephal./anneau nerveux } \\
\quad " \text { /pore excréteur } \\
\text { I. oerophage } \\
\text { L. queue } \\
\text { Oeufs }\end{array}$ & $\begin{array}{c}39-46 \\
0,87 \\
0,25-0,27 \\
0,6 \\
0,7-0,75 \\
9,0 \\
0,35 \\
0,035 \times 0,0125\end{array}$ & $\begin{array}{c}32-70 \\
0,8-1,5 \\
0,3-0,6 \\
0,6-0,9 \\
0,44-0,7 \\
6,8-12 \\
0,4-0,9 \\
0,03-0,042 x \\
0,0173-0,028\end{array}$ & $\begin{array}{l}54 \\
0,87 \\
0,32 \\
0,66 \\
0,835 \\
8,43 \\
0,705 \\
0,016=0,017 \times \\
0,011-0,013\end{array}$ \\
\hline
\end{tabular}

En 1928, JOYEUX, GENDRE et BAER signalent ce nématode comme assez fréquent (2 fois sur 11) chez Erythrocebus patos en Haute-Guinée, mais localisé d̀ l'intestin alors que notre prélèvement a été fait au niveaú de l'estomac.

Famille des Thelaziidoe RAILLIET, 1916.

Genre: Thelazla BOSC 1819.

Hôte: Melierax metabates HEUGLIN (faucon) (Kolda 5-7-64 dans l'œil) 2 femelies immatures de Thelazia dont la détermination spécifique est impossible.
Famille des Gnasthostomidoe RAILLIET, 1895.

Sous-Familie des Gnasthostominae, BAYLIS et LANE, 1920.

Genre: Tonqua BLANCHARD, 1904.

Espèce: Tonqua tiara (LINSTOW, 1879).

Hôte : Varanus niloticus L.

Le 13-12-64 Kolda, dans l'estomac 2 mâles et 1 femelle adultes.

Sans date, localisation : poumons (nous pensons qu'ıl s'agit plutôt de l'estomac qui a peutêtre été ouvert prématurément avant ou pen- 


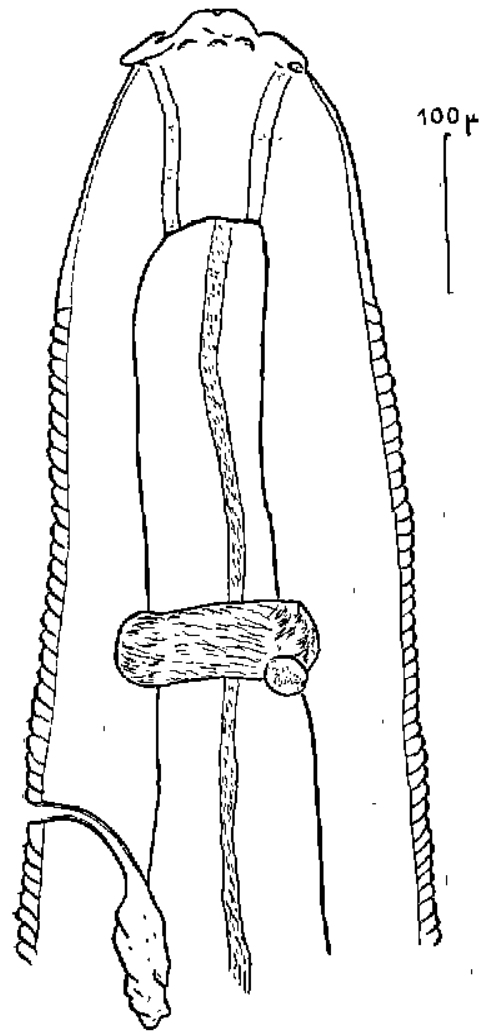

Fig. no 5. - Extrémité antérieure de la femelle de P. muricola récoltée chez une Syivicapra.

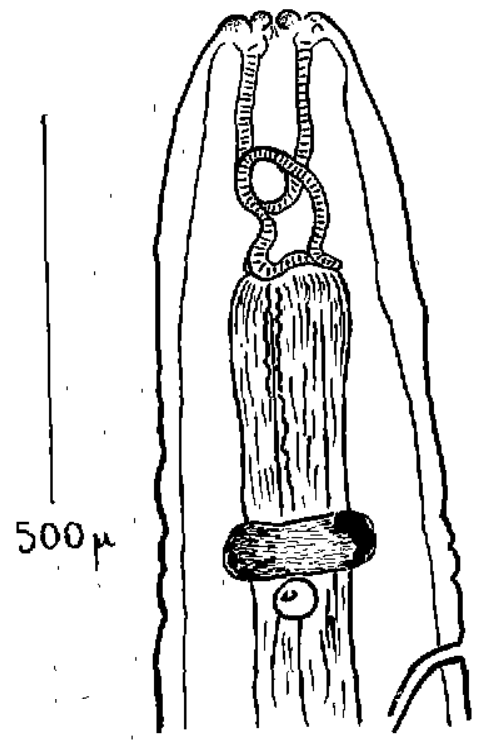

Fig. no 7. - Streptopharagus pigmentatus extrémilé antérieure d'un spécimen femelle récolté chez un Cercopithecus potas.

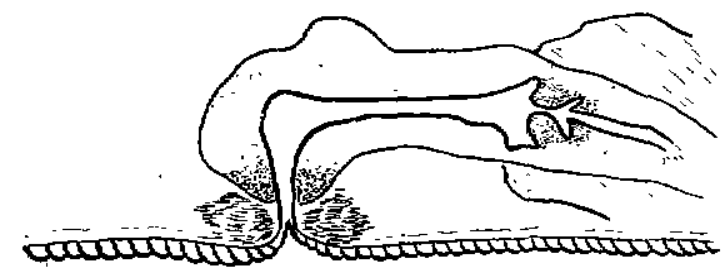

$500 \mu$

Fig. $n^{\circ} 6$. - Vulve el ovojecteur de la femelle de P. muri- cola récoltée chez Sylvicapra.

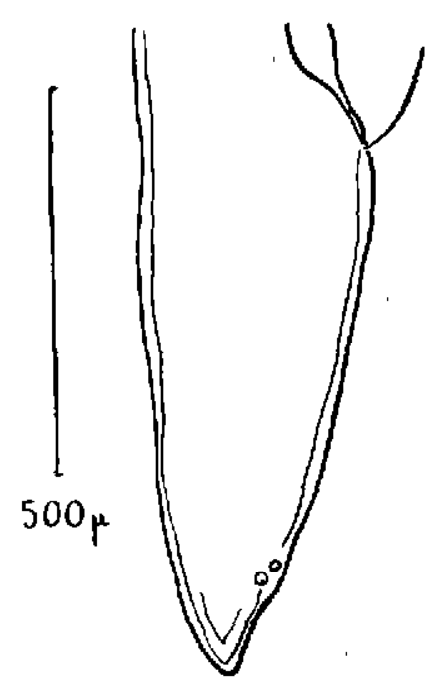

Fig. n० 8. - Extrémité postérieure de la femelle de 5 . pigmentotus de la fig. 7 . 
dant l'autopsie), 2 mâles et 15 femelles dont 3 immatures.

Cefte espèce est très commune chez les Varamidae au Sénégal aussi bien en Haufe-Casamance que dans la région de Dakar.

\section{SUPERFAMILLE DES FILARIOIDEA WEINLAND, 1858}

Famille des Filariidae CLAUS, 1885

Sous-Famille des Aproctinae YORKE et MAPLESTONE, 1926.

Genre Squamofilaria SCHMERLING, 1925.

Syn. : Coronofilaria YORKE et MAPLESTONE, 1926.

Syn. : Austrofilaria JOHNSTON et MAWSON, 1940 (d'après ANDERSON et CHABAUD, 1958).

Hôtes: Coracias naevius DAUDIN L. (Rollier varié),

une femelle Kolda 18-2-65,

une femelle Kolda 28-2-65.

Coracias abyssinica HERMANN (Rollier d'Abyssinie),

une femelle Kolda 28-3-64.

une femeile Kolda 5-3-65.

Ces quatre femelles provenant de quatre Coracias ont toutes été récoltées dans les tissus souscutanés.

Ne présentant aucune ornementation papillaire cuticulaire ( small insignifiant papillae scaftered irregularly » YORKE et MAPLESTONE, 1926) elles n'appartiennent pas à l'espèce $S$. pillersi.

Cependant en 1958, ANDERSON et CHABAUD font remarquer «qu'on ne peut se fier aux marques, cuticularres comme caractères génériques des filaires ». Et ces mêmes auteurs dans leur tableau dichotomique permettant la détermination des quatre espèces du genre : S. sicki (STRACHEN, 1957) S. coronato (RUD, 1809) S. vestibulata (JOHNSON et MAWSON, 1940 ) et $S$. pillersi (YORKE et MAPLESTONE, 1926) se servent uniquement des caractères du mâle (spicules, papilies caudales).

En l'absence de spécimens mâles, nous pouvons donc difficilement attribuer un nom d'espèce aux exemplaires que nous avons examinés.

Si l'on s'en réfère à l'hôte, nos spécimens pourraient être des femelles de S. coronota, cette espèce ayant toujours été récoltée sous la peau d'oiseaux du genre Coracias. Chez Coracias garrulus L. par RUDOLPHI (1809), KOROLIOWA (1926) en Russie, BOULENGER (1926) en Egypte ef BAYLIS (1939) en Angleterre ; chez Coracias bengalensis aux Indes par SINGH en 1949 et Chez Coracias abyssinica au Congo-Léopoldville par VUYILSTEKE en 1957.

Sous-Famille des Setariinae YORKE et MAPLESTONE, 1926.

Genre Hastospiculum.

Espèce : H. mocrophallos (PARONA, 1889).

Hôte : Voranus miloticus $\mathrm{L}$.

Le 1-1-64, 1 exemplaire femelle en mauvals état récolté sous la peau.

Le 1-1-64, 2 exemplaires femelles récoltés sous la peau.

Le 3-12-64, 15 exemplaires femelles dans le péritoine et la cavité thoracique.

Dans le dernier prélèvement, les vers traversent les muscles intercostaux ef s'insinuent entre les plans musculaires. Certains atteignent la région sous-cutanée. La plupart des spécimens sont en très mauvals état avec la cuticule déchirée, l'intestin ef les anses utérines plus ou moins dilacérés.

II semble, cependant, d'après l'observation des parties intactes et en se référant au travall de BAYLIS, 1930, que l'on puisse rapporter ces exemplaires à l'espèce Hostospiculum macrophallos (PARONA, 1889) qui d'après CHABAUD et ROUSSELOT (1956) serait très largement répandue des Indes jusqu'en Afrique tropicale (Brazzaville), et ces prélèvements le prouveraient, même en Afrique de l'Ouest.

\section{PENTASTOMIDA \\ Ordre Porocephalida Heymons}

Nettorhynctus (Armillifer) armillotus (WYMAN, 1845) formes nymphales.

Hôtes : " Cercopithecus aethiops var. sabaeus (L.) 》.

(Kolda ; 8-8-64; no 68) 15 nymphes enkystées dans le péritoine.

Hôte : Ichneumia albicauda Cuv. (Mangouste à queve bianche) (Kolda, no 114) 4 nymphes enkystées sous la peau.

Hôte : Ichneumia albicauda (Cuv., Kolda, rec. no 83). 
5 nymphes enkystées sur paroi péritonéale.

Le parasitisme par formes nymphales de Porocephalidae (porocéphalose) semble être extrêmement fréquent chez les Viverridae au Sénégal. NOC en 1922 signale un cas d'infestation massive chez Genetfo pardina Is. GEOFFROY de la région de Dakar.

Les formes adultes étant hébergées par des serpents, particulièrement des pythons (dans les sacs aériens), l'infestation des Viverridae ef des rongeurs s'explique par l'ingestion d'aliments souillés par des déjections de serpents parasités.

Le polyxénisme de ce pentastomide est tel que ses kystes nymphaux sont rencontrés chez un très grand nombre de mammifères y compris l'homme (NOC et NOGUE, 1919).

\section{RÉSUMÉ ET CONCLUSION}

L'étude d'un premier lot de parasites internes (Nematoda et Pentostoma) récoltés à l'autopsie de mammifères, oiseaux et reptiles de HauteCasamance (Sénégal) a permis de signaler :

Chez Varanus miloticus: Hastospiculum macrophallos (PARONA, 1889). Tonqua tiara (O. v. LINSTOW, 1879).

Chez Coracias naevius ef $C$. abyssinicus : un Squamofiları qui pourrait appartenır à l'espèce S. coronata (RUD. 1809).
Chez Mclierax metobates (faucon) : un Thelazia localisé à l'œil.

Chez Erythrocebus patos SCHREBER : Streptopharagus pigmentatus (O. $\quad$. LINSTOW, 1897).

Chez un singe vert, Cercopithecus aethiops var. sabaeus (L.) et sur Sylvicapra grimmia : Protospirura muricola GEDOELST, 1918.

Chez Cercopithecus aethiops var, saboeus ef Ichneumio albicauda Cuv. (mangouste à queue blanche) : les formes nymphales de Netiorhynchus (Armillifer) armillatus (WYMANN, 1845) (Pentastomo).

Un second lot de nématodes comportant notamment un certain nombre de Physaloptera parasites de l'estomac des singes et des petits carnivores ainsi' que des filaires d'oiseaux et de mammifères appartenant aux genres Diplotriaena RAILLIET et HENRY, 1909, Setoria WIBORG, 1795, Dirofilaria RAILLIET et HENRY, 1911 est en cours d'étude et fera l'objet d'une note ultérieure.

$$
\begin{gathered}
\text { Institut d'Elevage et de Médecine vétérinaire } \\
\text { des Pays Tropicaux. } \\
\text { Laboratoire national de l'Elevage } \\
\text { et de Recherches vétérinaires du Sénégal. } \\
\text { Dakar et Service des Grandes Endémies, } \\
\text { Kolda (Hte-Casamance). }
\end{gathered}
$$

\section{SUMMARY}

First note about some endoparasites of Upper Casamance (Senegal) wild animals

\footnotetext{
The study of an internai parasite first serie (Nematoda and Pentastoma) collecred during the Upper Casamance (Senegal) mammal, bird autopsy allowed to indicate :

In Varanus miloticus : Hastaspiculum macrophallos (Parona, 1889).

: Tanqua tiara (o. v. Linstow, 1879).

In Coracias naevius and C.abyssi- : A Squamofilorio that might belong to the nicus

: (Rud. 1809) S. coronata species.

In Melierax metabates (Falcon) : A Thelazia localized in its eye.

In Erythrocebus patos Schreber : Streptopharaqus pigmentatus (o. v. Linstow,

: 1897).

In a green monkey

: Protospirura muricolo Gedoelst, 1918.

Cercopithecus aethiops var. saba-

eus (L.) and in Sylvicopro grim- : mio
} 


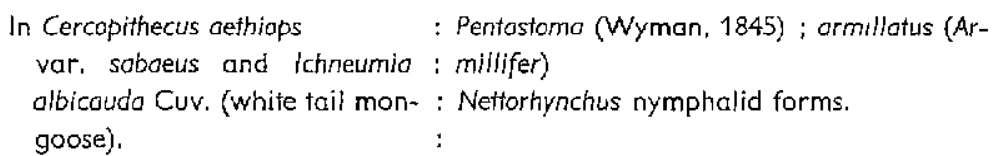

A second nematode serie including a number of Physaloptera, monkey and little carnivora stomach parasits, as also mammal and bird filarias belonging to, Diplotriaena, 1909, Railliet and Henry, Setaria, 1795, Wiborg, Dicrofilaria, 1911, Railliet and Henry is studying. A further work about them will be carried out.

\section{RESUMEN}

Primera nota sobre algunos endoparásitos de los animales salvajes de Alta-Casamancia (Senegal).

El estudio de una primera serie de parásitos iniernos (Nematoda y Pentastoma) recogidos durante la autopsia de mamíferos, pájaros y reptiles de Alta-Casamancia (Senegal) permitıó notar :

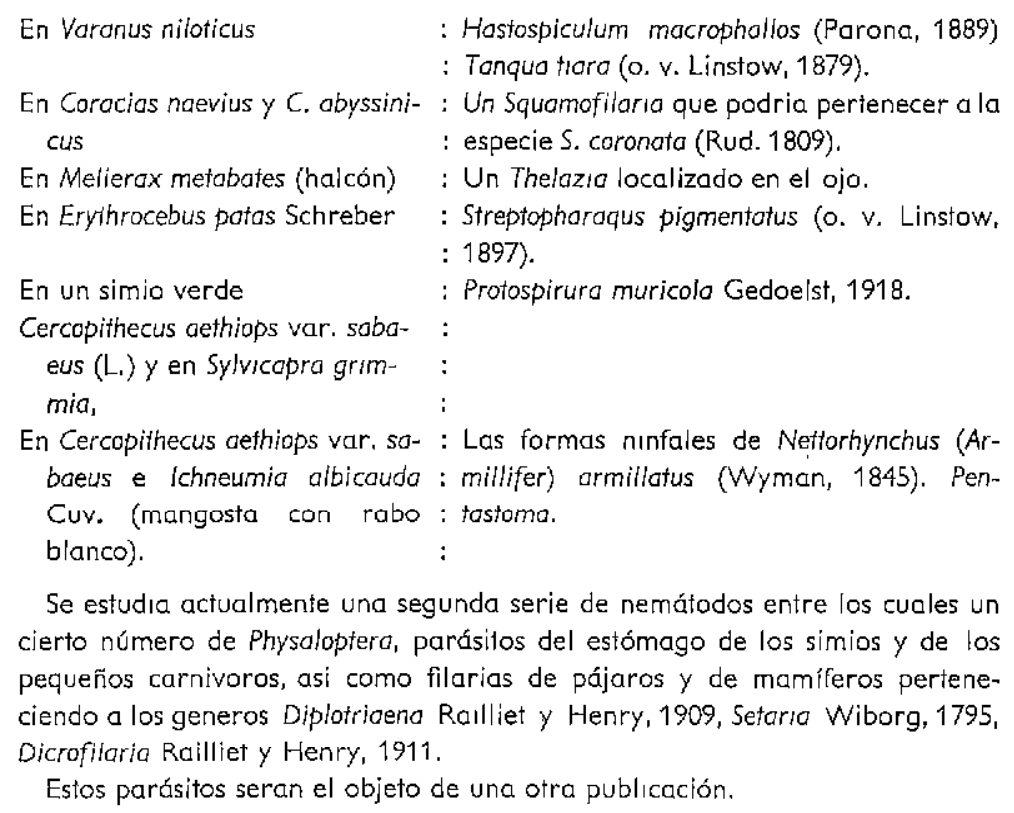

\section{BIBLIOGRAPHIE}

ANDERSON (R. C.) et CHABAUD (A. G.). Taxonomie de la filaire Squamofilaria sicki (Strachen, 1957) n. comb. ef place du genre Squamofilaria Schmerling, 1925 dans la sousfamille Aproctinae. Ann. Parasit, hum. comp. (1958), XXXIII, 3, $254: 266$.

BAYLIS ( $H$. A.). - The nematode genus Tanquo R. Blanchard. Ann. Mag. Not. Hist. (1916), ser, 8, 17, $223: 232$.

BAYLIS (H. A.). 一 On the nematode genus Strep- topharagus with some remarks on the genus Spirocerca. Trans, Roy. Soc, trop. med. hyg., (1923), 16,486: 497.

BAYLIS (H. A.). - On a collection of Nematodes from Nigeria mammals (chiefly Rodents). Parasitology (1928), XX, $280: 304$.

BAYLIS (H. A.). - Filaria macrophallos Parona, and the genus Hastopiculum Skrjabin (Nematoda). Ann. Mag. Nat. Hist. (1930) (Scr. 10), VI, $672: 677$. 
BAYLIS (H. A.). - Further records of parasitic worms from British vertebrates. Ann. Mag. Nat. Hist. (1939), 4, $473: 498$.

BLANC (G.). - - Un nématode nouveau (Streptopharagus armatus n.g. n. sp.) parasite du macaque. C. R. Soc. Biol. (1912), 74, 456 : 457.

BLANCHARD (R.). - Tonqua n. g., remplaçant Ctenocephalus von Linstow. Arch. Parasit. Paris (1904), VIll, 478.

BOULENGER (C. L.). - Report on a collection of parasitic nematodes, mainly from Egypt. Porositology, (1928), 20, $32: 55$.

CHABAUD (A. G.) et ROUSSELOT (R.). - Sur quelques filaires d'Afrique Equatoriale. Ann. Parosit. hum. comp. (1956), XXXI, 1/2, 53 : 98.

FOSTER (A. O.) et JOHNSTON (C.M.). - A preliminary note on the identity, life cycle and pathogenicity of an important nematode parasite of captive monkeys. Amer. J. Trop. Med. (1939), XIX, 265-877.

GEDOELST (L.). - Notes sur la faune parasitaire du Congo Belge. Rev. Zool. Afr. (Bruxelles), (1916-1918), V, $1: 90$.

JOYEUX (Ch.), GENDRE (E.) et BAER (J. G.). Recherches sur les Helminthes de l'Afrique Occidentale Française. Coliection de la Soc. Path. Exot. Monographie 11 (1928), 120 pages.

KOROLIOWA (A. M.). - Connaissance des Fildires chez les Oiseaux de la Russie. J. Gor. Inst. Vet. Med. (1926), Moscou, 3, $92: 110$ (Ouvrage non consulté).
MYERS (J.). - Helminth parasites of reptiles, birds and mammals in Egypt. Streptophoraqus kuntzi sp. nov. from rodents with a review of the genus. Conad. Z. Zool. (1954), 32, 366 : 374.

NOC (F.) et NOGUÉ. - Notes sur un cas de porocéphalose. Bull. Soc. Médico-Chirurg. Franç. Ouest-Afric. (1919), 1, 5, 6:9 (Anal. in Bull. Inst. Past. 1920, 288).

NOC (F.). - Infestation massive naturelle de la genetie du Sénégal par des larves de porocéphales. Bull. Soc. Path. Exot. (1922), $15,621: 631$.

$\operatorname{ORTLEPP}\left(R . J_{1}\right)$, - On a collection of Helminths from Dutch Guiana. J. of Helminth. (1924), $11,15: 40$.

SEURAT (L. G.). - Sur un nouveau spiroptère du chat ganté. C. R. Soc. Bıol. (1914), LXXV|l, $344: 347$.

SINGH (S. N.). - - Studies on the helminth parasites of birds in Hyderabad states. J. Helminth. (1949), 23, $39: 56$.

VUYLSTEKE (C.). - Nématodes parasites d'oiseaux. Exploration du Parc Notional Garumba, Mission H. de Saeger, (1957), 8, $3: 20$ (ouvrage non consulté).

YAMAGUTI (S.). - The nematodes of vertebrates (Systema helminthum) vol. III, 2 part. Interscience Publishers, Inc. N. Y. (1961), 1261 pages.

YORKE (W.) et MAPLESTONE (P. A.). - The Nematode parasites of vertebrates. Londres (1926), 536 pages. 